\title{
The Magnetic Flux Rope Structure of a Triangulated Solar Filament
}

\author{
Yang Guo ${ }^{1}\left(\mathbb{C}, \mathrm{Yu} \mathrm{Xu}{ }^{1}\right.$, M. D. Ding ${ }^{1}$, P. F. Chen ${ }^{1}$, Chun $\mathrm{Xia}^{2}$, and Rony Keppens ${ }^{3}$ (1) \\ ${ }^{1}$ School of Astronomy and Space Science and Key Laboratory for Modern Astronomy and Astrophysics, Nanjing University, Nanjing 210023, People's Republic of \\ China; guoyang@nju.edu.cn \\ ${ }^{2}$ School of Physics and Astronomy, Yunnan University, Kunming 650050, People's Republic of China \\ ${ }^{3}$ Centre for mathematical Plasma Astrophysics, Department of Mathematics, KU Leuven, Celestijnenlaan 200B, B-3001 Leuven, Belgium \\ Received 2019 August 20; revised 2019 September 12; accepted 2019 September 17; published 2019 October 3
}

\begin{abstract}
Solar magnetic flux ropes are core structures driving solar activities. We construct a magnetic flux rope for a filament/prominence observed at 01:11 UT on 2011 June 21 in AR 11236 with a combination of state-of-the-art methods, including triangulation from multiperspective observations, the flux rope embedding method, the regularized Biot-Savart laws, and the magnetofrictional method. First, the path of the filament is reconstructed via the triangulation with $304 \AA$ images observed by the Atmospheric Imaging Assembly on board Solar Dynamics Observatory $(S D O)$ and by the Extreme Ultraviolet Imager on board the twin Solar Terrestrial Relations Observatory. Then, a flux rope is constructed with the regularized Biot-Savart laws using the information of its axis. Next, it is embedded into a potential magnetic field computed from the photospheric radial magnetic field observed by the Helioseismic and Magnetic Imager on board SDO. The combined magnetic field is finally relaxed by the magnetofrictional method to reach a nonlinear force-free state. It is found that both models constructed by the regularized Biot-Savart laws and after the magnetofrictional relaxation coincide with the $304 \AA$ images. The distribution of magnetic dips coincides with part of the filament/prominence material, and the quasi-separatrix layers wrap the magnetic flux ropes, displaying hyperbolic flux tube structures. These models have the advantages of constructing magnetic flux ropes in the higher atmosphere and weak magnetic field regions, which could be used as initial conditions for magnetohydrodynamic simulations of coronal mass ejections.
\end{abstract}

Unified Astronomy Thesaurus concepts: Solar activity (1475); Solar filaments (1495); Solar prominences (1519); Solar photosphere (1518); Solar corona (1483); Solar magnetic fields (1503)

\section{Introduction}

Magnetic flux ropes (MFRs) are the core ingredient driving solar activities. The eruptions of MFRs usually lead to coronal mass ejections (CMEs) and flares (Forbes \& Isenberg 1991; Lin $\&$ Forbes 2000; Chen 2011; Cheng et al. 2014). MFRs are able to support solar filaments or prominences, particularly in the dips of the magnetic field lines (Aulanier \& Démoulin 1998; Guo et al. 2010; Mackay et al. 2010; Blokland \& Keppens 2011). To fully understand the dynamics of major solar activities, it is crucial to quantify the three-dimensional (3D) magnetic field distribution in the solar atmosphere. However, a magnetic field can only be accurately measured routinely on the photosphere. The magnetic field in the upper atmosphere is usually constructed by a physical model, such as the nonlinear force-free field (Wiegelmann \& Sakurai 2012; Guo et al. 2017a), magnetohydrostatic (Low \& Zhang 2004; Zhu et al. 2013, 2016), and magnetohydrodynamic (MHD; Kliem et al. 2013; Jiang et al. 2016; Amari et al. 2018; Inoue et al. 2018; Guo et al. 2019) models.

Nonlinear force-free field models have been widely applied to construct the 3D magnetic field with MFRs (e.g., Yan et al. 2001; Régnier \& Amari 2004; Canou et al. 2009; Guo et al. 2010; Jing et al. 2010; Inoue et al. 2012; Yang et al. 2015, 2016; Zhong et al. 2019). Some common features of these reconstructed MFRs are that they are always located near a strong magnetic field region (photospheric field strength stronger than a few hundred Gauss) and lie along the polarity inversion line at an altitude not much higher than the photosphere (forming bald patches if the local magnetic field is tangential to the photosphere). A few exceptions were presented in Jiang et al. (2014), Liu et al. (2016), and James et al. (2018), where they found MFRs either near a weak field region (photospheric field strength weaker than 100 Gauss) or at a high altitude above the photosphere with hyperbolic flux tubes. Most of the nonlinear force-free field methods in the aforementioned studies use a potential field as the initial state and the vector magnetic field observed on the photosphere as the boundary condition. Then, that initial state is relaxed to the final result. However, both a weak boundary magnetic field and high-lying flux rope structures may prevent a successful relaxation to the final flux rope structures.

A good way to overcome the drawbacks in reconstructing flux ropes in regions of weaker fields and higher altitudes is to incorporate the observed information in the corona. Malanushenko et al. $(2012,2014)$ implemented a modified Grad-Rubin method to include the volume information, namely, the torsional parameter $\alpha$ constrained by the coronal loop geometry. Dalmasse et al. (2019) developed a Data-Optimized Coronal Field Model framework, where infrared coronal polarimetry is used to constrain the coronal magnetic field models. van Ballegooijen (2004) proposed the flux rope insertion method to incorporate the information of a twisted flux rope in the corona directly. This method has been widely applied to model diffuse or decaying active regions and polar crown prominences (Bobra et al. 2008; Savcheva \& van Ballegooijen 2009; Su et al. 2009, 2015). There are a few free parameters in the flux rope insertion method, such as the path of a filament, the axial and poloidal magnetic fluxes, and the radius of the flux rope, which need to be further constrained by observations. Furthermore, the initially inserted flux rope is out of equilibrium and can be improved by a more physical model. Titov et al. (2014) addressed this equilibrium problem using an 
MFR embedding method, while the embedded MFR is restricted to a toroidal arc shape. Recently, Titov et al. (2018) removed this geometric limitation by proposing regularized Biot-Savart laws (RBSL) to model an MFR with an axis of arbitrary path and circular cross sections. The modeled flux rope is also approximately force-free and serves well as the initial condition to construct a nonlinear force-free field model.

In this Letter, we reconstruct the axis path of a flux rope from multiperspective observations by the Solar Dynamics Observatory (SDO; Pesnell et al. 2012) and by the twin Solar Terrestrial Relations Observatory (STEREO). Then, we embed a flux rope into a potential magnetic field (Titov et al. 2014) following the reconstructed axis path using RBSL (Titov et al. 2018). The initial state is further relaxed to a nonlinear forcefree field state by a magnetofrictional (MF) code (Guo et al. 2016a, 2016b). The observations and our modeling methods are described in Section 2. Results are presented in Section 3. We finally summarize and discuss our results in Section 4.

\section{Observations and Modeling Methods}

A filament eruption on 2011 June 21 in AR 11236 was observed by the Atmospheric Imaging Assembly (AIA; Lemen et al. 2012) on board $S D O$ and by the Extreme Ultraviolet Imager (EUVI; Wuelser et al. 2004) in the telescope package of the Sun Earth Connection Coronal and Heliospheric Investigation (Howard et al. 2008) on board both STEREO_A and B. Zhou et al. (2017) studied the 3D geometric structure and kinetic evolution of this erupting filament. Here, we study the 3D magnetic field. We embed a flux rope following the filament axis into a potential magnetic field to construct a combined non-potential magnetic field. The basic idea is similar to that in Titov et al. (2014), but the MFR is computed with the RBSL method (Titov et al. 2018), and we do not need to remove the background potential field in a channel region along the inserted flux rope axis as done by van Ballegooijen (2004). The combined non-potential field is further relaxed to a nonlinear force-free field state by the MF code (Guo et al. 2016a, 2016b). The details of the procedure are explicated as follows.

First, we use a set of three $304 \AA$ images at 01:11 UT to construct the $3 \mathrm{D}$ path of the filament axis, as done by Zhou et al. (2017). The separation angles between STEREO_A/B and $S D O$ are $96^{\circ} / 93^{\circ}$ (Figure $1(\mathrm{a})$ ); thus the perspectives of the spacecraft constitute a favorable configuration for triangulation (Thompson 2009). The $304 \AA$ images observed by all three instruments are employed to construct both the western and eastern parts of the filament axis, as shown by the green and cyan circles in Figures 1(b)-(d).

Second, we prepare a projection-corrected vector magnetic field, as shown in Figures 2(a) and (b). The vector magnetic field was observed by the Helioseismic and Magnetic Imager (HMI; Scherrer et al. 2012; Schou et al. 2012; Hoeksema et al. 2014) on board SDO. The data series name is "hmi.B_720s." The rotation matrix used to correct the projection effect can be found in Guo et al. (2017a). We note that the reference longitude and latitude for the projection are those of the middle point of the two filament footprints, W5.6 N14.2. The projection-corrected vector magnetic field is also preprocessed to remove the Lorentz force and torque (Wiegelmann et al. 2006).
Third, we set the physical parameters for RBSL. There are four free parameters, the path of the filament axis, $\mathcal{C}$, the minor radius of the flux rope, $a$, the magnetic flux, $F$, and the electric current, I. The filament path $\mathcal{C}$ has been derived by triangulation of the STEREO_A/B and SDO $304 \AA$ images and overlaid on the vertical magnetic field as shown in Figure 2(a). Because the RBSL method requires a closed path for the electric current circuit in the whole space and a zero normal component of the magnetic field $B_{z 1}$ on the bottom boundary outside the MFR footprints, we adopt a mirror image of $\mathcal{C}$ to close the path. The flux rope radius is constrained by the filament width. As measured by Zhou et al. (2017), the halfwidth of the filament is about $0.01 R_{\odot}$, but the authors noted that this quantity is not well constrained and the filament material often does not fill the whole MFR. Here, we use twice the filament half-width as the minor radius of the MFR, namely, $a=0.02 R_{\odot}$. The peripheries of the flux rope at the two footprints of the filament are overplotted on the vertical magnetic field (Figure 2(a)). We measure the vertical magnetic flux at the two footprints and find that $F_{+}=0.4 \times 10^{20} \mathrm{Mx}$ and $F_{-}=-7.0 \times 10^{20} \mathrm{Mx}$. The magnetic flux of the flux rope is assumed to be the average of the unsigned values, i.e., $F=\left(\left|F_{+}\right|+\left|F_{-}\right|\right) / 2=3.7 \times 10^{20} \mathrm{Mx}$. Then, the electric current is calculated following Equation (12) in Titov et al. (2018): $I=(5 \sqrt{2} F) /\left(3 \mu_{0} a\right)=5.0 \times 10^{11} \mathrm{~A}$, where $\mu_{0}$ is the vacuum permeability. We note that $I$ could also be negative, which corresponds to a negative magnetic helicity. In our case, the magnetic field chirality is sinistral, namely, the axial field points to the left if one stands on the positive polarity to observe the filament axis. And the projected shape of the filament axis has a forward-S shape. Both results indicate that the magnetic field possesses a positive magnetic helicity.

Fourth, we embed the MFR derived by the RBSL method with the aforementioned physical parameters into a potential field. We compute the potential field using the Message Passing Interface Adaptive Mesh Refinement Versatile Advection Code (MPI-AMRVAC; Keppens et al. 2003, 2012; Porth et al. 2014; Xia et al. 2018). To keep the observed and processed normal magnetic field, $B_{z 0}$, unchanged after linearly combining the MFR field and potential field, we draw two circles delineating the footprints of the MFR (the green circles in Figure 2(a)) within which the normal magnetic field, $B_{z 1}$, computed by the RBSL method is subtracted from $B_{z 0}$. The subtracted magnetic field, $B_{z 0}-B_{z 1}$, is adopted as the bottom boundary for computing the potential field. Then, the MFR is added to the potential magnetic field, and the combined magnetic field is shown in Figure 2(c), which is named model 1 hereafter.

Finally, we use model 1 in Figure 2(c) as the initial condition and the vector magnetic field in Figure 2(b) as the bottom boundary condition to do an MF relaxation (Guo et al. 2016a, 2016b). The final result as shown in Figure 2(d) is named model 2 . We summarize the definitions of models 1 and 2 as follows:

1. Model 1: the magnetic field constructed by the MFR embedding method. An MFR field is embedded into a potential magnetic field that is computed with the subtracted normal magnetic field, $B_{z 0}-B_{z 1}$, on the bottom boundary.

2. Model 2: the magnetic field relaxed from model 1 with the observed and preprocessed vector magnetic field as the boundary condition. 

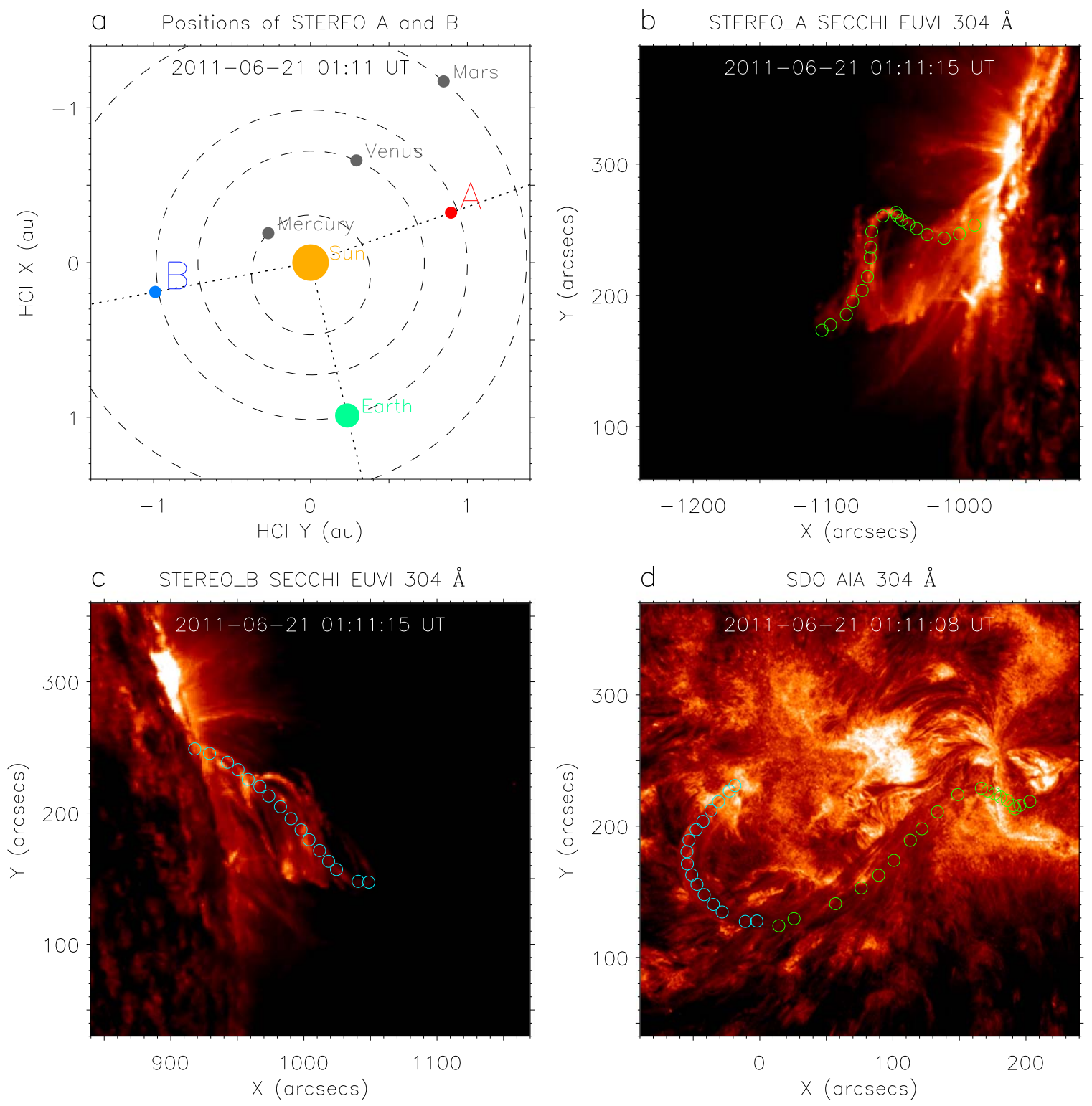

Figure 1. (a) Positions of STEREO_A and $B$ at 01:11 UT on 2011 June 21 in the Heliocentric Inertial (HCI) coordinate system. (b)-(d) Open circles showing the 3D positions of the filament axis, overlaid on the $304 \AA$ images observed by STEREO_A/EUVI, STEREO_B/EUVI, and SDO/AIA. The green circles are measured by STEREO_A/EUVI and SDO/AIA, while the cyan circles are measured by STEREO_B/EUVI and SDO/AIA.

We use two metrics, namely the current-weighted average of the sine of the angle between the current and the magnetic field, $\sigma_{J}$, and the average of the absolute change of the fractional magnetic flux, $\left\langle\left|f_{i}\right|\right\rangle$, to quantify the force-free and divergencefree conditions, respectively. We find that $\sigma_{J}=0.56$ and $\left\langle\left|f_{i}\right|\right\rangle=1.9 \times 10^{-5}$ for model 1 , and $\sigma_{J}=0.31$ and $\left\langle\left|f_{i}\right|\right\rangle=3.2 \times 10^{-4}$ for model 2 . The force-free condition has been improved and the divergence-free error is kept within an acceptable level. The force-free metric, $\sigma_{J}=0.31$, corresponds to an average angle of 18.1 between the magnetic field and electric current. This value is close to, although not as low as, the best case applied to observations (e.g., Guo et al. 2016a).

\section{Results}

To quantify the difference between model 1 constructed by the MFR embedding method (Figure 2(c)) and model 2 after the MF relaxation (Figure 2(d)), we compare the flux ropes with the $304 \AA$ images from different viewing angles, in terms of their morphologies and magnetic dips. We also compute the quasi-separatrix layers (QSLs) around the flux ropes, twist numbers, and magnetic helicity of the flux ropes.

The magnetic field vectors and coordinates in Figures 2(c) and (d) can be back-projected to the viewing angles of $S T E R E O \_A / B$ or $S D O$ by the rotation matrix $\mathcal{R}_{x}\left(-B_{0}\right) \mathcal{R}_{y}(-L) \mathcal{R}_{x}\left(B_{1}\right)$, where $\mathcal{R}_{x}$ is the elementary rotation matrix around the $x$-axis, $\mathcal{R}_{y}$ the elementary rotation matrix around the $y$-axis, $B_{0}$ the latitude of the disk center, $L$ the longitude of the reference point in the vector magnetic field, and $B_{1}$ the latitude of the reference point. We note that $L$ is measured in reference to the local central meridian of STEREO_A/B or SDO. The coordinates of $\left(B_{0}, L, B_{1}\right)$ for STEREO $\_A / B$ and $S D O$ are $\left(6^{\circ} .9,-90^{\circ} .9,14^{\circ} .2\right),\left(-7^{\circ} .2,98^{\circ} .1\right.$, $\left.14^{\circ} .2\right)$, and $\left(1 \circ 7,5 \circ 6,14^{\circ} .2\right)$, respectively.

The back-projected magnetic field lines are overlaid on the $304 \AA$ images for both models 1 and 2 in all three viewing angles by STEREO_A/B and SDO (Figure 3). It can be seen that 

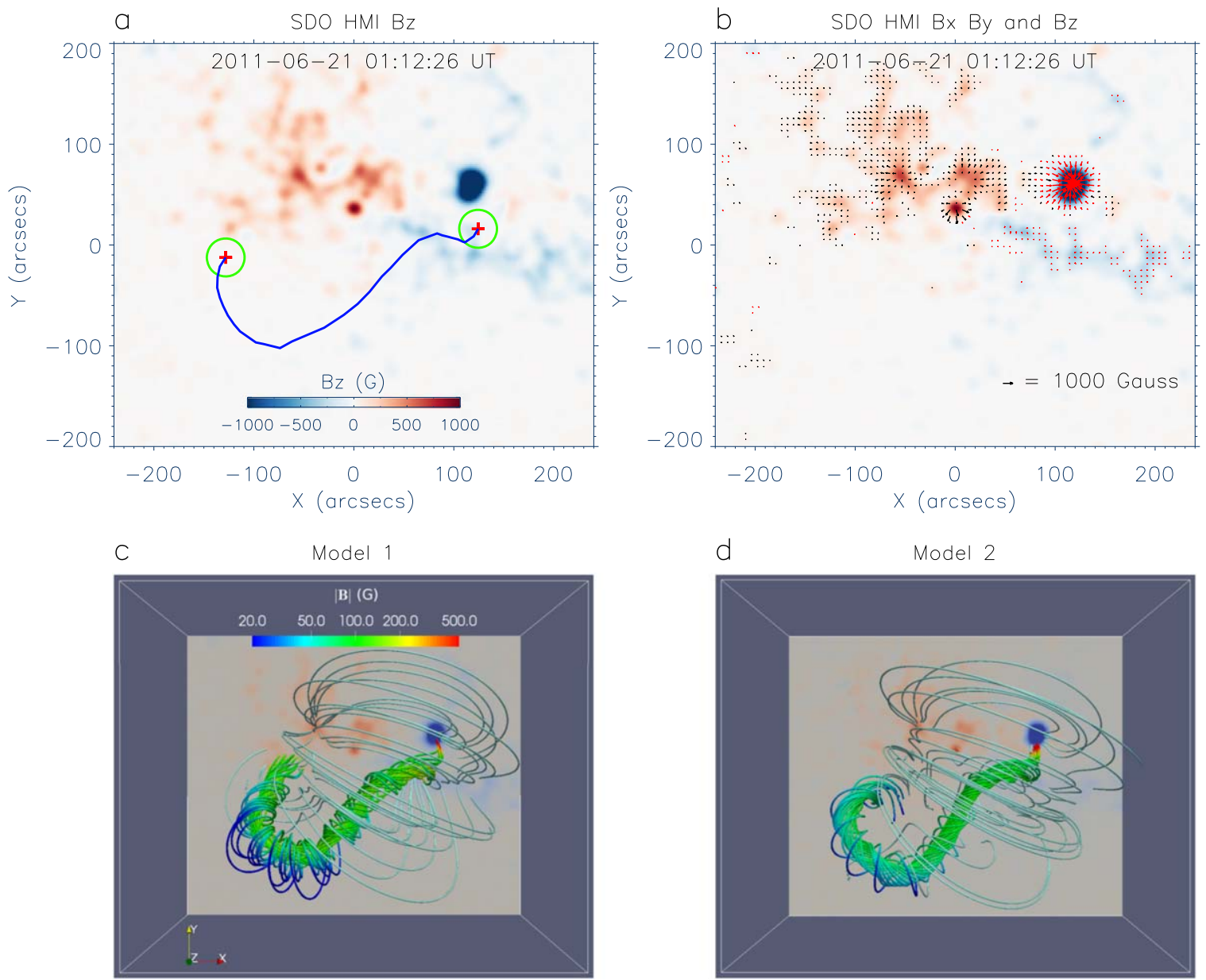

Figure 2. (a) $S D O / \mathrm{HMI}$ vertical magnetic field, $B_{z}$, at 01:12 UT on 2011 June 21 . The blue solid line illustrates the path of the filament axis, the red plus signs indicate the two footprints of the filament axis, and the green circles mark the periphery of the filament footprints. The magnetic field has been projected into a local coordinate system, where the $z=0$ plane is tangential to the solar surface at (W5.6, N14.2), the middle point of the two footprints of the filament, and the $z$-axis points to the radial direction of the Sun. (b) SDO/HMI horizontal magnetic field, $B_{x}$ and $B_{y}$, overlaid on $B_{z}$. (c) The flux rope constructed by the MFR embedding method. (d) The MFR further relaxed by the MF method.

in general the shape of the flux rope mimics the morphology of the filament/prominence. The most prominent features are the elbows in Figures 3(a) and (d) and the long axis path in Figures 3(b) and (e). As shown in Figure 1(b), the axis makes a turn at $(X, Y)=\left(-1050^{\prime \prime}, 260^{\prime \prime}\right)$ to form an elbow there. This feature is clearly reproduced by both models in Figures 3(a) and (d). While in Figures 3(b) and (e), the long axis path is also well covered by the field lines of the flux ropes in the two models.

There are also some subtle differences in the flux ropes constructed by the two models. From all the panels in Figure 3, we find that the flux rope after the MF relaxation (model 2) leans more toward the south than that derived by the MFR embedding method (model 1). The western part of the flux rope is lower in model 2 than that in model 1 , as shown in Figures 3(a), (c), (d), and (f). Also, the eastern footpoint of the flux rope moves to the south in model 2, as shown in Figures 3(c) and (f).

Magnetic dips are thought to have a close relationship with filament material. They are the positions where the magnetic field satisfies the following two equations: $(\boldsymbol{B} \cdot \nabla) B_{z}>0$ and $B_{z}=0$ (Titov et al. 1993). Here, the coordinate system is chosen as the local Cartesian coordinates, where the $z$-axis is along the local vertical direction. Once we know a magnetic field model, we first search for the places where the equation $B_{z}=0$ is satisfied. It is a linear equation with one unknown if the magnetic field is derived by trilinear interpolation. Then, the positions of the magnetic dips are found by further requiring $(\boldsymbol{B} \cdot \nabla) B_{z}>0$. We integrate a series of field line segments, which are about $1.1 \times 10^{4} \mathrm{~km}$ long and centered at the magnetic dips. The coordinates of the field line segments are rotated to different viewing angles of STEREO_A/B and $S D O$ as shown in Figure 4.

The distribution of magnetic dips shows some spatial correspondence with the filament/prominence. For example, the magnetic dips generally cover the filament axis path, as shown in Figures 4(b) and (e). The upper part of the magnetic dips coincides with the prominence, as seen by STEREO_B in Figures 4(c) and (f). We also notice some discrepancies between the magnetic dips and the filament/prominence. Magnetic dips are absent in the elbow part of the prominence, as shown in Figures 4(a) and (d). There is also a region in the south with magnetic dips, where little filament/prominence material is seen, as shown in all panels of Figure 4. Filament/ prominence material could flow along a magnetic field line without dips (Wang 1999; Karpen et al. 2001), or not enough material may get accumulated in the corona even if there are 
a

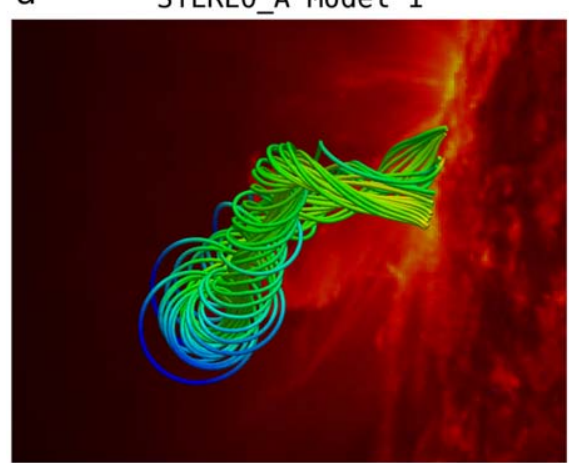

b

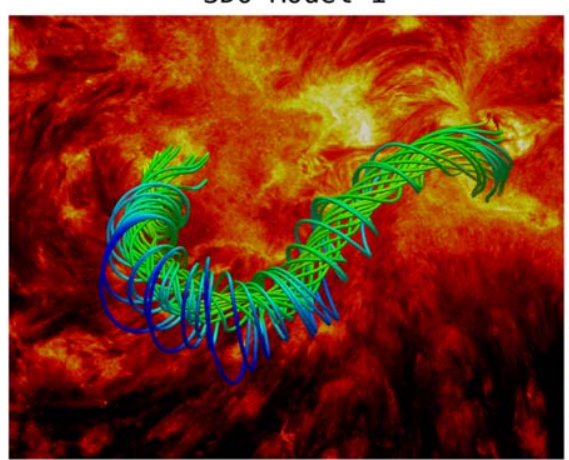

C

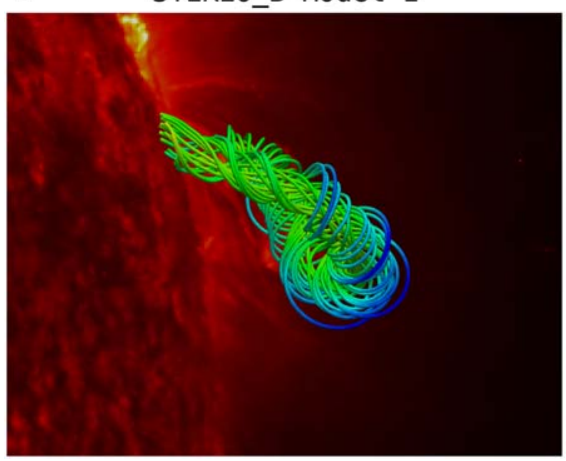

d

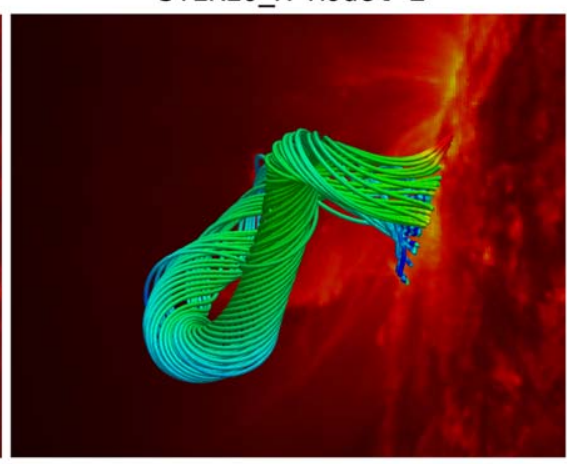

e

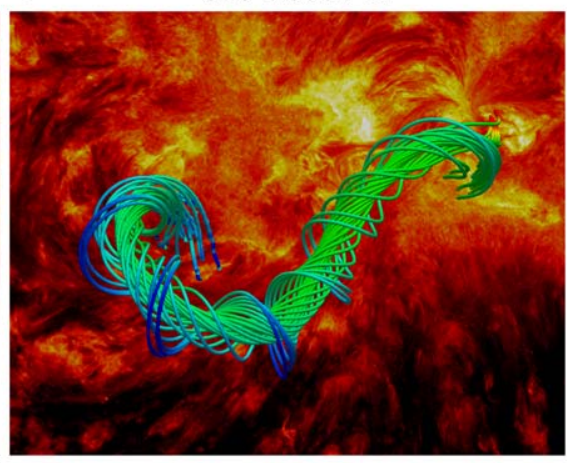

$f$

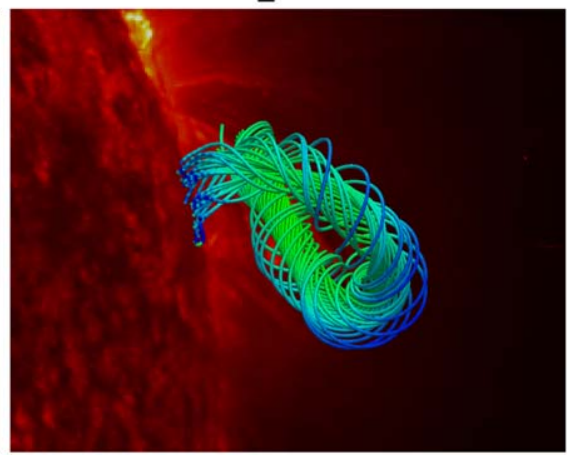

Figure 3. (a) MFR constructed by the MFR embedding method, overlaid on the $304 \AA$ image, which was observed by STEREO_A/EUVI at 01:11 UT on 2011 June 21. The viewing angle is from STEREO_A. (b) Same as (a) but viewed from SDO. (c) Same as (a) but viewed from STEREO_B. (d) MFR further relaxed by the MF method, overlaid on the same background as (a). (e) Same as (d) but viewed from SDO. (f) Same as (d) but viewed from STEREO_B.

magnetic dips, which might explain both aforementioned features.

QSLs provide the geometric boundary of an MFR and the possible locations for magnetic reconnection. We compute the QSLs around the flux rope on two selected slices for the two models using a method proposed by Pariat \& Démoulin (2012) and implemented by Yang et al. (2015). Figure 5(a) shows that, for the magnetic field derived from the MFR embedding method, the flux rope is wrapped by a QSL, and the wrapped QSL forms a hyperbolic flux tube. This structure can still be found in the model after the magnetofrictional relaxation (Figure 5(c)), although the two bifurcated QSL sections below the flux rope revealed in Figure 5(a) are not so obviously seen in Figure 5(c), where the QSL sections merge into one with complicated internal structures. QSLs on another slice for the two models in Figures 5(b) and (d) show similar shapes, where the flux rope is fully surrounded by the QSL. The QSL bifurcates from the right side and extends to a lower height.
Finally, we calculate the twist numbers and magnetic helicity of the MFRs in both models 1 and 2. Here, the boundary of the flux rope is defined as the inner QSL in Figures 5(b) and (d). The axis is defined as the field line that is most perpendicular to this selected slice, as shown in Figures 5(b) and (d). The formula to calculate the twist is given by Berger \& Prior (2006), and an example can be found in Guo et al. (2017b). It is found that the twist is 4.3 turns for the flux rope in model 1, and it is 3.9 turns for that after the MF relaxation (model 2). The magnetic helicity is calculated with $\mathcal{H}=\mathcal{T} F^{2}$, where $F$ is the magnetic flux within the QSL that surrounds the flux rope. We find that $F_{1}=4.3 \times 10^{20} \mathrm{Mx}$ for model 1 and $F_{2}=4.6 \times 10^{20} \mathrm{Mx}$ for model 2 . Both values are different from the initial value of $3.7 \times 10^{20} \mathrm{Mx}$, which is estimated from the observations. Thus, the magnetic helicity is derived as $\mathcal{H}_{1}=8.0 \times 10^{41} \mathrm{Mx}^{2}$ and $\mathcal{H}_{2}=8.3 \times 10^{41} \mathrm{Mx}^{2}$. Note that the writhe is omitted in calculating the magnetic helicity. The writhe of the filament axis was estimated to be 0.1 in a follow- 

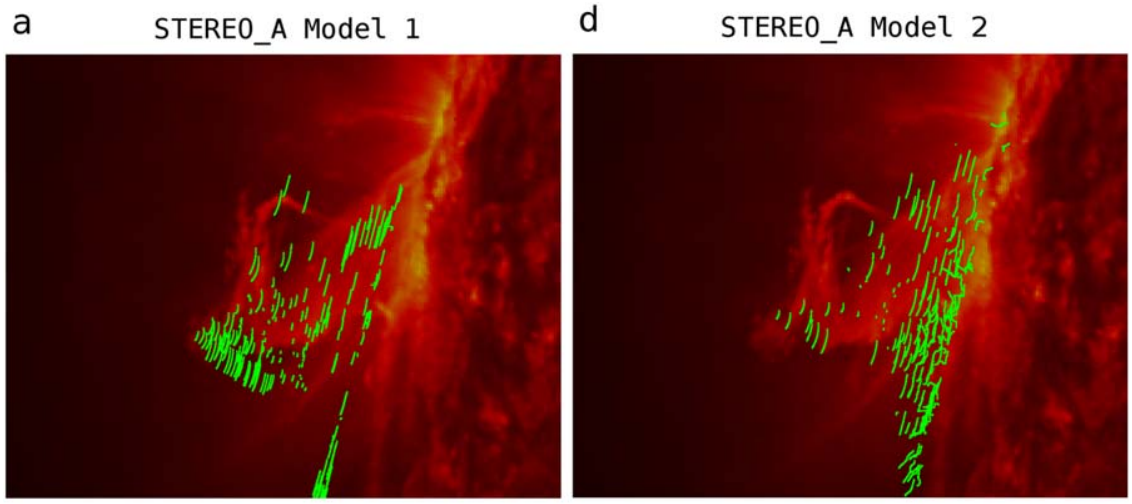

b

SDO Model 1

e
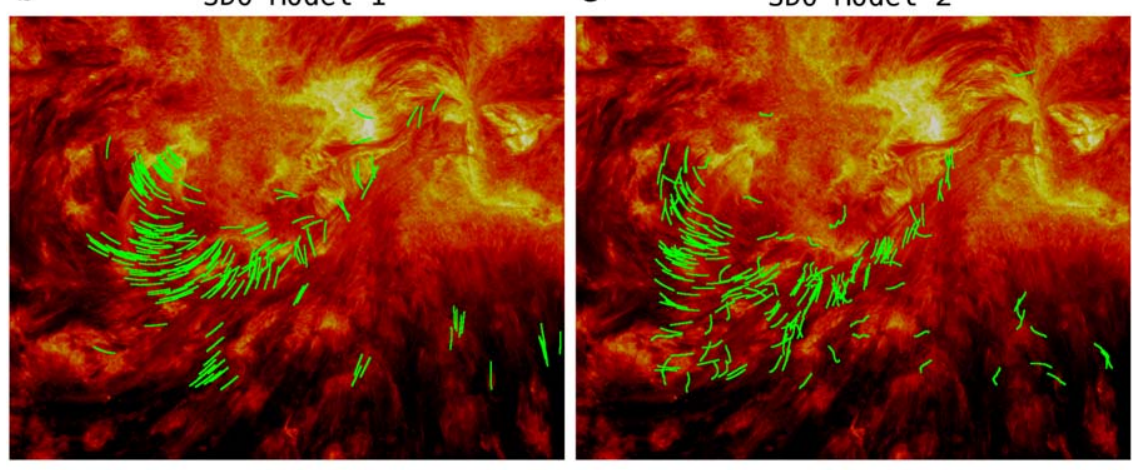

C

STERE0 B Model 1

f

STEREO_B Model 2
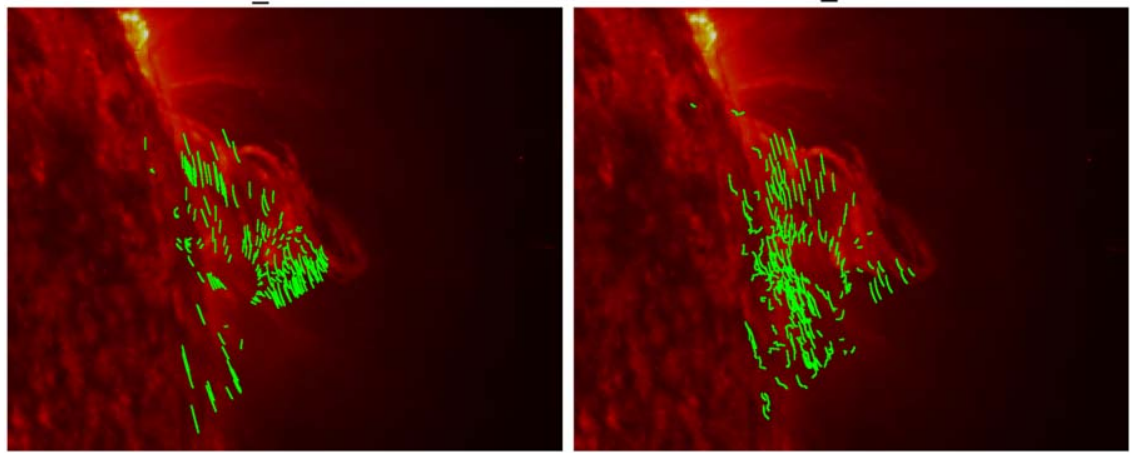

Figure 4. (a) Magnetic dips computed from the MFR constructed by the MFR embedding method overlaid on the $304 \AA$ image, which was observed by $S T E R E O \_A$ / EUVI at 01:11 UT on 2011 June 21. The viewing angle is from STEREO_A. (b) Same as (a) but viewed from SDO. (c) Same as (a) but viewed from STEREO_B. (d) Magnetic dips computed from the MFR further relaxed by the MF method, overlaid on the same background as (a). (e) Same as (d) but viewed from SDO. (f) Same as (d) but viewed from STEREO_B.

up study by us, which is much less than the twist numbers obtained here.

\section{Summary and Discussion}

We construct an MFR model for the filament/prominence observed at 01:11 UT on 2011 June 21 with a procedure combining triangulation (Thompson 2009) using the STEREO_A/B and SDO multiperspective observations, the MFR embedding method (Titov et al. 2014), the RBSL method (Titov et al. 2018), and the MF code (Guo et al. 2016a, 2016b). The filament/prominence axis path is determined by the $304 \AA$ images observed by STEREO_A/EUVI, STEREO_B/ EUVI, and SDO/AIA. Then, the path is used as one of the key parameters to construct a twisted flux rope by the RBSL method. The flux rope is embedded into a potential field, which is computed from the radial magnetic field observed by SDO/ HMI. The derived magnetic field of the flux rope embedded in the potential model can be further relaxed by the MF code to attain a better nonlinear force-free field model.

We quantitatively compare some physical parameters of the two models derived by the MFR embedding method (model 1) and by the further MF relaxation (model 2). Model 1 has a lower (and thus better) divergence-free metric, while model 2 has a lower (and thus better) force-free metric. The twist of the flux rope in model 1 is 4.3 turns, which is slightly larger than the 3.9 turns in model 2 . The magnetic helicity shows a similar value, with $\mathcal{H}_{1}=8.0 \times 10^{41} \mathrm{Mx}^{2}$ and $\mathcal{H}_{2}=8.3 \times 10^{41} \mathrm{Mx}^{2}$. The results imply that the MF relaxation could somewhat reduce the twist of an MFR. We also note that the magnetic fluxes in both models 1 and 2 are larger than the prescribed value. These excess magnetic fluxes are supposed to come from the potential magnetic field, as we artificially reduce the potential magnetic field at the footprints of the MFR, which has 


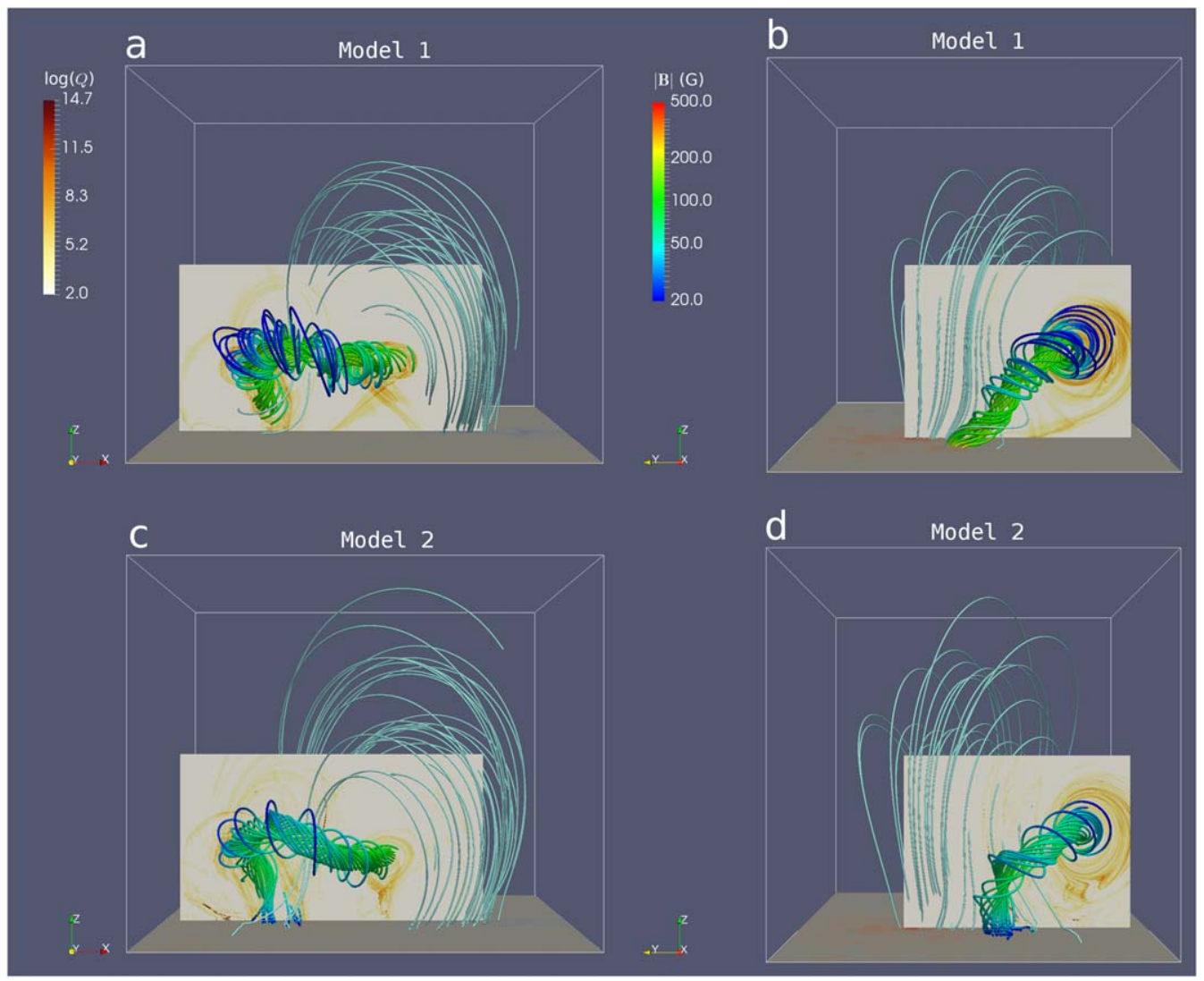

Figure 5. (a) QSLs computed from the MFR constructed by the MFR embedding method, shown in a slice perpendicular to the $y$-axis. (b) QSLs computed from the same model as (a) but on a slice perpendicular to the $x$-axis. (c) Same as (a) but for the MFR further relaxed by the MF method. (d) Same as (b) but for the MFR further relaxed by the MF method.

a non-zero contribution to the magnetic field structure in the body of the MFR.

The MFRs in models 1 and 2 are compared with multiperspective observations. In general, they coincide with the observed filament/prominence. However, the flux rope in model 2 is deformed a little after the relaxation. Here, we note that the boundary condition used in model 2 is not fully consistent with the RBSL model, which assumes circular cross sections with a uniform radius that implies an equal magnetic field strength. However, the observations show that the magnetic field strengths at the two footprints are different, where $\left|F_{+}\right|=0.4 \times 10^{20} \mathrm{Mx}$ at the positive footprint is much smaller than $\left|F_{-}\right|=7.0 \times 10^{20} \mathrm{Mx}$ at the negative footprint, even with the same area. The magnetic dips from both models 1 and 2 overlap with a major part of the observed filament/ prominence material. In addition, we find hyperbolic flux tube structures in the QSLs of both models.

The filament/prominence was observed at 01:11 UT on 2011 June 21 high up in the corona. The maximum heights of the axes in models 1 and 2 are about 81 and $87 \mathrm{Mm}$, respectively, and the minimum magnetic field strength along the axes is about 66 and 23 Gauss, respectively. Can we use a nonlinear force-free field model to construct its magnetic structure? Zhou et al. (2017) have studied the time profiles of height and velocity during the eruption of the filament/ prominence. They showed that the velocity is almost zero at 01:11 UT (see Figure 5 in Zhou et al. 2017). Therefore, a nonlinear force-free field model is valid for this moment. The MFR embedding method together with the RBSL and MF methods have the advantage of constructing MFRs in the high atmosphere and weak magnetic field regions. Observations show that many CMEs originate from quiet-Sun regions and high up in the corona (Chen 2011), where the traditional nonlinear force-free field extrapolation methods might fail to construct MFRs. However, the MFR embedding method together with the RBSL and MF methods could still obtain such quiescent MFRs. So, they could be applied to construct MFRs for quiescent prominence and coronal cavity systems. We also look forward to using these models as initial conditions for data-driven or data-constrained MHD simulations in the future.

The SDO data are available courtesy of NASA/SDO and the AIA and HMI science teams. The SECCHI data are available courtesy of STEREO and the SECCHI consortium. Y.G. and Y. $X$. thank Zhenjun Zhou for his help on the tie pointing method to reconstruct 3D filament axis. Y.G., Y.X., M.D.D., and P.F. C. are supported by NSFC (11773016, 11733003, 11533005, and 11961131002). C.X. is supported by NSFC (11803031). R. $\mathrm{K}$. is supported by the ERC advanced grant PROMINENT, and by the joint FWO-NSFC project G0E9619N. The numerical calculations in this paper have been done using the computing facilities of the High Performance Computing Center (HPCC) in Nanjing University.

\section{ORCID iDs}

Yang Guo (iD https://orcid.org/0000-0002-9293-8439

Rony Keppens (D) https://orcid.org/0000-0003-3544-2733 


\section{References}

Amari, T., Canou, A., Aly, J.-J., Delyon, F., \& Alauzet, F. 2018, Natur, 554,211

Aulanier, G., \& Démoulin, P. 1998, A\&A, 329, 1125

Berger, M. A., \& Prior, C. 2006, JPhA, 39, 8321

Blokland, J. W. S., \& Keppens, R. 2011, A\&A, 532, A93

Bobra, M. G., van Ballegooijen, A. A., \& DeLuca, E. E. 2008, ApJ, 672, 1209

Canou, A., Amari, T., Bommier, V., et al. 2009, ApJL, 693, L27

Chen, P. F. 2011, LRSP, 8, 1

Cheng, X., Ding, M. D., Guo, Y., et al. 2014, ApJ, 780, 28

Dalmasse, K., Savcheva, A., Gibson, S. E., et al. 2019, ApJ, 877, 111

Forbes, T. G., \& Isenberg, P. A. 1991, ApJ, 373, 294

Guo, Y., Cheng, X., \& Ding, M. 2017a, ScChE, 60, 1408

Guo, Y., Pariat, E., Valori, G., et al. 2017b, ApJ, 840, 40

Guo, Y., Schmieder, B., Démoulin, P., et al. 2010, ApJ, 714, 343

Guo, Y., Xia, C., \& Keppens, R. 2016a, ApJ, 828, 83

Guo, Y., Xia, C., Keppens, R., Ding, M. D., \& Chen, P. F. 2019, ApJL, 870, L21

Guo, Y., Xia, C., Keppens, R., \& Valori, G. 2016b, ApJ, 828, 82

Hoeksema, J. T., Liu, Y., Hayashi, K., et al. 2014, SoPh, 289, 3483

Howard, R. A., Moses, J. D., Vourlidas, A., et al. 2008, SSRv, 136, 67

Inoue, S., Kusano, K., Büchner, J., \& Skála, J. 2018, NatCo, 9, 174

Inoue, S., Shiota, D., Yamamoto, T. T., et al. 2012, ApJ, 760, 17

James, A. W., Valori, G., Green, L. M., et al. 2018, ApJL, 855, L16

Jiang, C., Wu, S. T., Feng, X., \& Hu, Q. 2014, ApJL, 786, L16

Jiang, C., Wu, S. T., Feng, X., \& Hu, Q. 2016, NatCo, 7, 11522

Jing, J., Yuan, Y., Wiegelmann, T., et al. 2010, ApJL, 719, L56

Karpen, J. T., Antiochos, S. K., Hohensee, M., Klimchuk, J. A., \& MacNeice, P. J. 2001, ApJL, 553, L85

Keppens, R., Meliani, Z., van Marle, A. J., et al. 2012, JCoPh, 231, 718

Keppens, R., Nool, M., Tóth, G., \& Goedbloed, J. P. 2003, CoPhC, 153, 317

Kliem, B., Su, Y. N., van Ballegooijen, A. A., \& DeLuca, E. E. 2013, ApJ, 779,129

Lemen, J. R., Title, A. M., Akin, D. J., et al. 2012, SoPh, 275, 17

Lin, J., \& Forbes, T. G. 2000, JGR, 105, 2375
Liu, R., Kliem, B., Titov, V. S., et al. 2016, ApJ, 818, 148

Low, B. C., \& Zhang, M. 2004, ApJ, 609, 1098

Mackay, D. H., Karpen, J. T., Ballester, J. L., Schmieder, B., \& Aulanier, G. 2010, SSRv, 151, 333

Malanushenko, A., Schrijver, C. J., DeRosa, M. L., \& Wheatland, M. S. 2014, ApJ, 783, 102

Malanushenko, A., Schrijver, C. J., DeRosa, M. L., Wheatland, M. S., \& Gilchrist, S. A. 2012, ApJ, 756, 153

Pariat, E., \& Démoulin, P. 2012, A\&A, 541, A78

Pesnell, W. D., Thompson, B. J., \& Chamberlin, P. C. 2012, SoPh, 275, 3

Porth, O., Xia, C., Hendrix, T., Moschou, S. P., \& Keppens, R. 2014, ApJS, 214,4

Régnier, S., \& Amari, T. 2004, A\&A, 425, 345

Savcheva, A., \& van Ballegooijen, A. 2009, ApJ, 703, 1766

Scherrer, P. H., Schou, J., Bush, R. I., et al. 2012, SoPh, 275, 207

Schou, J., Scherrer, P. H., Bush, R. I., et al. 2012, SoPh, 275, 229

$\mathrm{Su}$, Y., van Ballegooijen, A., McCauley, P., et al. 2015, ApJ, 807, 144

Su, Y., van Ballegooijen, A., Schmieder, B., et al. 2009, ApJ, 704, 341

Thompson, W. T. 2009, Icar, 200, 351

Titov, V. S., Downs, C., Mikić, Z., et al. 2018, ApJL, 852, L21

Titov, V. S., Priest, E. R., \& Demoulin, P. 1993, A\&A, 276, 564

Titov, V. S., Török, T., Mikic, Z., \& Linker, J. A. 2014, ApJ, 790, 163

van Ballegooijen, A. A. 2004, ApJ, 612, 519

Wang, Y. M. 1999, ApJL, 520, L71

Wiegelmann, T., Inhester, B., \& Sakurai, T. 2006, SoPh, 233, 215

Wiegelmann, T., \& Sakurai, T. 2012, LRSP, 9, 5

Wuelser, J.-P., Lemen, J. R., Tarbell, T. D., et al. 2004, Proc. SPIE, 5171, 111

Xia, C., Teunissen, J., El Mellah, I., Chané, E., \& Keppens, R. 2018, ApJS, 234, 30

Yan, Y., Deng, Y., Karlický, M., et al. 2001, ApJL, 551, L115

Yang, K., Guo, Y., \& Ding, M. D. 2015, ApJ, 806, 171

Yang, K., Guo, Y., \& Ding, M. D. 2016, ApJ, 824, 148

Zhong, Z., Guo, Y., Ding, M. D., Fang, C., \& Hao, Q. 2019, ApJ, 871, 105

Zhou, Z., Zhang, J., Wang, Y., Liu, R., \& Chintzoglou, G. 2017, ApJ, 851, 133

Zhu, X. S., Wang, H. N., Du, Z. L., \& Fan, Y. L. 2013, ApJ, 768, 119

Zhu, X. S., Wang, H. N., Du, Z. L., \& He, H. 2016, ApJ, 826, 51 